\title{
Dificultades de la Lógica Deóntica: el Problema de las Tareas Abstractas ${ }^{1}$
}

\author{
Miguel López Astorga²
}

\begin{abstract}
RESUMEN: La propuesta relativa a que existe una lógica deóntica con la que los seres humanos operan en algunos dominios tiene que solucionar el problema de los discretos resultados de las tareas de selección deónticas abstractas. En una investigación reciente, se ha tratado de resolver este problema argumentando que las tareas de selección deónticas abstractas suelen ir acompańadas de instrucciones ambiguas que no son fáciles de interpretar y planteando a participantes nuevas versiones de esas mismas tareas con instrucciones más simples y menos equívocas. En este trabajo, analizamos los textos que se utilizan en tal investigación y los resultados que se obtienen en ella y llegamos a la conclusión de que, realmente, no soluciona los problemas de las tareas deónticas abstractas iniciales. Desde nuestro punto de vista, las respuestas de sus participantes en las nuevas versiones deberían haber sido más óptimas y existen fenómenos pragmáticos y relacionados con la comprensión de los textos que pueden haber influido en sus resultados experimentales.
\end{abstract}

PALABRAS ClAVE: Comprensión de textos. Lógica deóntica. Pragmática. Razonamiento condicional. Tarea de selección.

\section{INTRODUCCIÓN}

La idea de que los seres humanos contamos con una lógica especial para realizar inferencias oportunamente en contextos o dominios deónticos, esto es, la idea de que contamos con una lógica deóntica, parece requerir, para su aceptación, que previamente se resuelva un problema relacionado con la tarea de selección de las cuatro tarjetas (WASON, 1966, 1968). Dicho problema reside en el hecho de que las versiones deónticas abstractas de la mencionada tarea, si bien arrojan resultados aceptables, tales resultados no alcanzan los porcentajes de ejecución correcta de las versiones deónticas de esa misma tarea con contenido

\footnotetext{
${ }^{1}$ Este artículo es resultado del proyecto $\mathrm{N}^{\circ} 1120007$, "El procesamiento de enunciados condicionales en los estudiantes de Educación Media: un estudio a partir de la tarea de selección de Peter Wason”, del Fondo Nacional de Desarrollo Científico y Tecnológico (FONDECYT), de la Comisión Nacional de Investigación Científica y Tecnológica (CONICYT), Ministerio de Educación, Gobierno de Chile. El autor, que es el investigador responsable del mencionado proyecto, agradece al programa FONDECYT $\mathrm{y}$ a las instituciones nombradas el financiamiento de este trabajo.

${ }^{2}$ Dr. en Lógica y Filosofía de la Ciencia. Instituto de Estudios Humanísticos “Juan Ignacio Molina” Universidad de Talca - milopez@utalca.cl
} 
temático concreto. Esta circunstancia es un problema porque cabe pensar que el motivo por el que en las versiones con contenido concreto se obtienen mejores resultados no es que, al enfrentarse a ellas, el participante opere en virtud de una lógica deóntica, sino que el contenido introduce algún factor adicional que facilita la ejecución, como, por ejemplo, por hacer referencia a una explicación cualquiera al respecto no basada en la tesis de la existencia de una lógica deóntica, una estructura costo-beneficio en la que es necesario localizar infractores, esto es, individuos que obtienen el beneficio y no pagan el costo. Tal es, como es sabido, básicamente, una de las tesis fundamentales de la teoría de los contratos sociales (COSMIDES, 1989; COSMIDES; TOOBY, 1992; FIDDICK et al., 2000; FIDDICK; ERLICH, 2010).

No obstante, Beller y Bender (2012) han tratado de salvar este inconveniente. Su tesis es que las versiones deónticas abstractas de la tarea de selección reflejan una ejecución más limitada o pobre que las versiones deónticas concretas porque las versiones deónticas abstractas con las que se ha trabajado hasta el momento adolecen de diversos defectos en los textos de sus instrucciones que dificultan la labor de los participantes. Desde su óptica, corrigiendo tales defectos, las tareas deónticas abstractas pueden aumentar significativamente su número de respuestas correctas.

Desde nuestro punto de vista, empero, Beller y Bender (2012) no logran lo que se proponen, ya que, por una parte, los resultados que obtienen con versiones deónticas abstractas corregidas según sus supuestos no son tan concluyentes como ellos los presentan y, por otra parte, no tienen en cuenta la influencia que pueden ejercer en la interpretación, por parte de los participantes, de los textos que acompañan a sus instrucciones diversas variables y fenómenos que, a menudo, inciden en los procesamientos de información que realizan los sujetos cuando se enfrentan a mensajes lingüísticos. A explicar en qué nos basamos para plantear estas afirmaciones dedicamos estas páginas. Así, comenzamos describiendo las características generales de las versiones deónticas abstractas y cuál es su problemática exacta con respecto a las versiones deónticas concretas. A continuación, exponemos las tesis de Beller y Bender (2012) y los problemas que, desde su óptica, han acompañado a las versiones abstractas utilizadas en la literatura. Con posterioridad, comentamos de manera detenida en qué consistió exactamente el trabajo experimental de Beller y Bender (2012). Finalmente, revisamos críticamente sus conclusiones experimentales y la interpretación que hacen de sus resultados e indicamos los aspectos que pueden cuestionarse en su investigación, las características que 
ésta debería haber tenido para poder aceptar sus conclusiones sin reservas y por qué no se pueden considerar los porcentajes que obtienen como definitivos o decisivos.

\section{LAS VERSIONES DEÓNTICAS ABSTRACTAS Y CONCRETAS DE LA TAREA DE SELECCIÓN}

Las dificultades que, en general, envuelven a la tarea de selección de las cuatro tarjetas de Peter Wason se encuentran relacionadas con el hecho de que, a pesar de tratarse de un ejercicio de razonamiento condicional sin la mayor complejidad, en sus versiones iniciales, la mayor parte de los participantes no responde correctamente. Tales versiones iniciales eran muy abstractas. En ellas, se presentaba una regla que establecía una relación condicional entre dos términos, una vocal y un número par, y se le mostraban al participante cuatro tarjetas -una con una vocal, otra con una consonante, otra con un número par y la última con un número impar- con el propósito de que indicara cuál o cuáles había que girar para determinar si la regla condicional era verdadera o falsa. Se notó, no obstante, que, cuando se introducían algunas modificaciones en las instrucciones, los resultados mejoraban de manera evidente. Sin embargo, como no todas las modificaciones posibles conducían a un mayor número de selecciones válidas, se inició todo un debate en el que se encuentra inserta la problemática que aquí se va a abordar. Ante el hecho de que eliminar el nivel de abstracción de la tarea y recurrir a contenido temático concreto no aseguraba necesariamente una ejecución más óptima de la tarea, una de las hipótesis que se defendió apuntaba a que la clave para aumentar el número de respuestas correctas residía en redactar las instrucciones en términos deónticos, pero un nuevo problema surgió cuando se comprobó que, como se ha señalado en la introducción, las tareas deónticas solían ir acompañadas de mejores resultados si incluían contenidos concretos.

Probablemente, la versión deóntica abstracta de la tarea de selección más representativa es la que se encuentra recogida en Cheng y Holyoak (1985) y que Beller y Bender (2012) comentan. En ella, el participante tiene que suponer que es una autoridad responsable de supervisar si las personas obedecen ciertas regulaciones que tienen, todas ellas, la misma forma general:

Si alguien realiza la acción $A$, entonces tiene primero que satisfacer la precondición $P$. 
Igualmente, se le especifica al participante que lo que la regla anterior establece es que, para que se le permita a alguien hacer $A$, primero tiene que haber cumplido requisito $P$. De la misma manera, se le muestran cuatro tarjetas y se le indica que contienen información sobre personas, señalando, cada una de ellas, por un lado, si una persona ejecutó o no la acción $A$ y, por el otro, si ese mismo individuo cumplió o no la precondición $P$. La labor del participante consiste, entonces, en comprobar si una regla determinada está siendo seguida o no y, así, en seleccionar la tarjeta o las tarjetas que debe girar para ello, eligiendo únicamente aquellas (o aquella) que sean (o sea) necesarias (o necesaria) para tener completa seguridad. Lo que se puede observar en las tarjetas es lo siguiente:

\section{Tarjeta (1) Ha realizado la acción $A$. \\ Tarjeta (2) No ha realizado la acción $A$. \\ Tarjeta (3) Ha cumplido la precondición P. \\ Tarjeta (4) No ha cumplido la precondición P.}

La respuesta es obvia si tenemos en cuenta que la regla es un enunciado condicional y que, según las prescripciones de la lógica de proposiciones, un enunciado condicional sólo es falso cuando su antecedente es verdadero y su consecuente es falso, esto es, para la forma general expuesta más arriba, en el caso de que se realice la acción $A$ y no se satisfaga la precondición $P$. Beller y Bender (2012) lo explican comentando que la regla se sigue cuando quien realiza la acción $A$ cumple la precondición $P$ y que, por tanto, es necesario girar dos tarjetas: la tarjeta (1) (Ha realizado la acción $A$ ), para revisar si esa persona ha cumplido la precondición $P$, y la tarjeta (4) (No ha cumplido la precondición $P$ ), para excluir la posibilidad de que esa persona haya realizado la acción $A$.

En opinión de Beller y Bender (2012), que la tarea sea tan abstracta no debería ser un problema desde la óptica de la teoría del esquema pragmático de razonamiento de Cheng y Holyoak (1985), que, lo tenemos que recordar, también se encuentra esbozada en trabajos como Cheng y Holyoak (1989), pues, para esta teoría, las personas poseen esquemas abstractos para operar con reglas de este tipo y que les permiten extraer las conclusiones adecuadas en contextos de acciones y precondiciones para realizarlas. Sin embargo, parece que existe una dificultad con respecto a este punto, ya que, como hemos indicado, las versiones deónticas de la tarea de selección con contenido concreto suelen ser ejecutadas de manera más favorable. Una versión deóntica 
concreta puede ser, por ejemplo, la del famoso problema de la edad para beber (COX; GRIGGS, 1982), al que también hacen referencia Beller y Bender (2012). En esta versión, la regla es semejante a la siguiente:

Si una persona bebe cerveza, entonces esa persona tiene que ser mayor de 16 años.

Las tarjetas, evidentemente, corresponden igualmente aquí a personas, pero ahora muestran, por una cara, si esa persona bebe o no cerveza y, por la otra, si esa misma persona es mayor de 16 años o no. Esta versión posee la misma estructura que la versión abstracta y la respuesta correcta es, por tanto, también obvia, pues las tarjetas que es preciso girar ante este escenario para comprobar si la regla es cumplida o no son la correspondiente a la persona que bebe cerveza (puede tener menos de 16 ańos) y la relativa a la persona menor de 16 años (puede estar tomando cerveza).

El problema aquí es, reiteramos, que los resultados en las versiones deónticas concretas similares a ésta suelen ser significativamente mejores que los de las versiones deónticas abstractas. Por ejemplo, por ceñirnos exclusivamente a datos a los que nos remiten Beller y Bender (2012), en Beller (2010) se nos informa de que un porcentaje de un $73,8 \%$ de respuestas correctas en versiones deónticas concretas de la tarea de selección puede descender a un 58,4\% de elecciones válidas en versiones deónticas abstractas de esa misma tarea. Sin duda, se puede pensar que un porcentaje del $58,4 \%$ no es un porcentaje tan bajo. No obstante, la tesis de que existe una lógica especial con la que el ser humano opera ante situaciones deónticas y los supuestos de la teoría del esquema pragmático de razonamiento (CHENG; HOLYOAK, 1985, 1989) exigen que no se registre tal diferencia de porcentajes entre las versiones concretas y las abstractas, pues, en definitiva, si los seres humanos contamos con los recursos mentales formales precisos para solucionar exitosamente problemas de índole deóntica, tales recursos deberían ser igualmente eficientes tanto en escenarios concretos como en escenarios abstractos.

Beller y Bender (2012), empero, argumentan que los resultados en las versiones deónticas abstractas de la tarea de selección son tan discretos porque tales versiones van acompańadas de una serie de dificultades que se pueden eliminar fácilmente, lo que puede llevarnos a porcentajes de elección correcta de tarjetas más próximos a los de las versiones concretas. Revisamos 
sus argumentos y los problemas que, desde su óptica, se dan en las versiones deónticas abstractas de la tarea en el apartado siguiente.

\section{Dificultades ObSERVADAS EN LA TAREA DEÓNTICA ABSTRACTA ORIGINAL}

Las dificultades que, a juicio de Beller y Bender (2012), se hallan presentes en las versiones deónticas abstractas similares a la inicial propuesta por Cheng y Holyoak (1985) son las siguientes:

A. Se indica que todas las normas o regulaciones responden a una misma forma general, lo que sugiere que no hay que revisar simplemente una regla, sino un conjunto de reglas probablemente diferentes.

B. La formulación de la regla en inglés es un poco equívoca. Dicha formulación en el texto original es ésta: "If one is to take action $A$, then one must first satisfy precondition P" (CHENG; HOLYOAK, 1985, p. 403). Nosotros hemos traducido aquí este enunciado como si alguien realiza la acción $A$, entonces tiene primero que satisfacer la precondición $P$. Sin embargo, según Beller y Bender (2012), la expresión If one is to take action $A$ es problemática porque puede entenderse que, en realidad, hace referencia a una intención, esto es, a "If someone wants to take action A..." (BELLER; BENDER, 2012, p. 115), que puede traducirse como si alguien quiere realizar la acción $A$, cuando lo que verdaderamente se encuentra condicionado deónticamente por la regla no es la intención, sino el hecho de realizar la acción $A$.

C. Hay una dimensión temporal establecida confusamente en la regla. La precondición $P$ debe cumplirse antes de realizar la acción $A$, pero la regla menciona a los dos elementos en el orden inverso, esto es, menciona antes a la acción $A$ que a la precondición $P$.

D. La precondición $P$ es presentada como necesaria, pues se utiliza el término must (tener que), sin especificarse si $P$ es una condición suficiente para la realización de la acción $A$.

E. Se solicita comprobar si una determinada regla está siendo seguida o no, lo que se expresa en el texto original de este modo: "to check that a certain regulation is being followed" (CHENG; HOLYOAK, 1985, p. 403), mientras que lo que se pregunta en versiones concretas como la del problema de la edad para beber es si la regla está siendo violada o no. Esta distinción no es sutil para Beller y Bender (2012), pues, para 
saber si la regla está siendo seguida o no, basta con girar la tarjeta (1) de la versión abstracta (Ha realizado la acción $A$ ). Seguir la regla sólo es importante para las personas a las que se les aplica, esto es, para las personas que realizan la acción $A$, por lo que únicamente parece necesario girar la tarjeta (1) para comprobar si esas personas han cumplido la precondición $P$. No obstante, el caso de la tarjeta (4) de la versión abstracta (No ha cumplido la precondición $P$ ) es diferente. Si esa persona ha realizado la acción $A$, efectivamente, ha violado la regla. Empero, si no la ha realizado, la regla, simplemente, no se le aplica. Así, ante las instrucciones de la versión deóntica abstracta de Cheng y Holyoak (1985), los participantes pueden preferir seleccionar exclusivamente la tarjeta (1).

F. Un último aspecto problemático del texto de Cheng y Holyoak (1985) es esta indicación: "Turn over only those [cards] that you need to check to be sure” (CHENG; HOLYOAK, 1985, p. 403), la cual podemos traducir como Gira sólo las tarjetas que necesitas comprobar para estar seguro. Esta indicación no es adecuada, a juicio de Beller y Bender (2012), porque puede provocar que los participantes sean excesivamente prudentes y piensen que no deben seleccionar demasiadas tarjetas. $\mathrm{Y}$ es que algunos pueden considerar que es suficiente hallar a un individuo que no sigue (o que viola) la regla.

De esta manera, desde el punto de vista de Beller y Bender (2012), la formulación de la versión abstracta original de Cheng y Holyoak (1985) torna compleja la labor de extraer la información deóntica auténticamente relevante en ella, induciendo a elegir solamente pocas tarjetas y a preferir especialmente la tarjeta (1). Estos factores, por sí mismos, explican, en su opinión, las disparidades de porcentajes de respuesta correcta entre las versiones deónticas abstractas y concretas y, siendo controlados y eliminando las dificultades propias de las versiones abstractas, los porcentajes deberían acercarse. Esto es precisamente lo que tratan de demostrar con sus tres experimentos. A continuación, los describimos y comentamos sus resultados.

\section{LOS TRES EXPERIMENTOS DE BELLER Y BENDER}

En cada uno de los tres experimentos de Beller y Bender (2012) se compara la tarea original de Cheng y Holyoak (1985) con cuatro nuevas versiones. Con estas nuevas versiones tratan de evitar los problemas descritos 
en el punto precedente. Todas ellas obedecen al mismo esquema general y presentan instrucciones similares, diferenciándose básicamente en la redacción de su regla. Nuestra traducción de las instrucciones generales que acompañan a las nuevas versiones es la siguiente:

Imagina que perteneces a una autoridad que comprueba si las personas cumplen o violan una regla determinada. La regla es: [una de las que se van a indicar más adelante]. Las tarjetas presentadas más abajo representan cuatro personas. Por un lado de cada tarjeta está escrito si la respectiva persona realiza o no la acción A y por el otro lado si cumple o no la condición P. Tu tarea: indica todas las tarjetas que tienes que girar-todas aquellas para las que necesites conocer la información que hay en su reverso-para comprobar si la respectiva persona viola la regla.

Persona (1) Realiza la acción A.

Persona (2) No realiza la acción $A$.

Persona (3) Cumple la condición P.

Persona (4) No cumple la condición P.

Es importante señalar que, aunque ello no se percibe en nuestra traducción, Beller y Bender (2012) modifican también en su texto todas las expresiones que, a su juicio, pueden dar lugar a una interpretación en términos de intención y las sustituyen por expresiones que remiten a la realización efectiva de la acción. Por lo demás, en el Experimento 1, las cuatro reglas usadas fueron:

Obligación O1: Si una persona realiza la acción A, entonces tiene que cumplir la condición $P$.

Liberación R1: Si una persona no realiza la acción A, entonces no necesita cumplir la condición P.

Prohibición B1: Si una persona no cumple la condición P, entonces no tiene que realizar la acción $A$.

Permiso P1: Si una persona cumple la condición P, entonces puede realizar la acción $A$.

Las reglas reciben las denominaciones $O 1, R 1, B 1$ y $P 1$ en función de las iniciales de los términos correspondientes en inglés (obligation, release, ban y permission). El número 1 , por su parte, indica que se trata del primer experimento. Lo relevante aquí es que $O 1$ es análoga a la regla original, que dos reglas están expresadas de un modo deóntico fuerte y describen una restricción deóntica explícitamente $(O 1$ y $B 1)$ y que las otras dos están expresadas de 
un modo deóntico débil y, por ello, no pueden ser violadas en un sentido deóntico, al no expresar una restricción deóntica explícitamente (R1 y P1).

En el Experimento 2, a las reglas se les ańade una sentencia adicional para que al participante se le haga más evidente el sentido de la prohibición que encierran. Las reglas son las siguientes:

Obligación O2: Si una persona realiza la acción A, entonces tiene que cumplir la condición P; de otra manera, no necesita cumplirla.

Liberación R2: Si una persona no realiza la acción A, entonces no necesita cumplir la condición $P$; de otra manera, tiene que cumplirla. Prohibición B2: Si una persona no cumple la condición P, entonces no tiene que realizar la acción $A$; de otra manera, puede realizarla.

Permiso P2: Si una persona cumple la condición P, entonces puede realizar la acción $A$; de otra manera, no tiene que realizarla.

En el Experimento 3, se reemplazaron las negaciones explícitas por verbos semánticamente equivalentes con el fin de eliminar otra dificultad potencial, esto es, la complejidad que entrańa el procesamiento de las negaciones explícitas. Nuestra traducción de las reglas de este experimento es ésta:

Obligación O3: Si una persona realiza la acción A, entonces está obligada a cumplir la condición P; de otra manera, está liberada de cumplirla.

Liberación R3: Si una persona no realiza la acción $A$, entonces está liberada de cumplir la condición $P$; de otra manera, está obligada a cumplirla.

Prohibición B3: Si una persona no cumple la condición P, entonces la acción A está prohibida; de otra manera, la acción A está permitida. Permiso P3: Si una persona cumple la condición P, entonces la acción A está permitida; de otra manera, la acción A está prohibida.

Beller y Bender (2012) creen que con estas nuevas tareas se puede esperar que la mayoría de los participantes seleccione las tarjetas correspondientes a la realización de la acción $A$ y al no cumplimiento de la condición $P$, esto es, las tarjetas correctas. Así, prescindiendo de detalles y centrándonos en los aspectos de la investigación de Beller y Bender (2012) con más repercusiones para este trabajo, podemos decir que interpretaron que sus participantes, estudiantes de la Universidad de Friburgo sin experiencia previa con versiones de la tarea de selección de las cuatro tarjetas, procedieron de tal manera que confirmaron 
sus predicciones y sus hipótesis, pues, desde su punto de vista, en la versión abstracta inicial de Cheng y Holyoak (1985) predominó la tendencia a elegir exclusivamente la tarjeta relativa a la realización de la acción $A$ y en las nuevas versiones se mejoró en este aspecto y se pudo observar una frecuencia mayor de selección de la anterior tarjeta junto con la referente al no cumplimiento de la condición $P$.

A nuestro juicio, sin embargo, esto es bastante discutible en dos sentidos. Por una parte, sus resultados no son tan óptimos como consideran y, por otra, los incrementos en los porcentajes de selección correcta que revelan pueden atribuirse a causas diferentes a la utilización de una lógica especial para extraer conclusiones en dominios deónticos, por lo que no logran el objetivo que se proponen y no queda demostrada de manera concluyente la existencia de una lógica deóntica en la mente humana. Argumentamos estas afirmaciones en el apartado siguiente.

\section{INCERTIDUMBRE CON RESPECTO A LA EXISTENCIA DE UNA LÓGICA DEÓNTICA}

Um problema metodológico que, de entrada, puede observarse en el trabajo de Beller y Bender (2012) es que no notan que modificar la redacción de las instrucciones y de las reglas de distintas versiones de la tarea de selección no es un modo de proceder que necesariamente nos ayude a demostrar nuestras hipótesis iniciales con respecto al razonamiento humano, ya que el incremento en los porcentajes de respuesta válida siempre puede ser interpretado como el efecto de factores lingüísticos, semánticos o pragmáticos asociados a la nueva redacción y a los nuevos términos empleados. Sin embargo, al margen de esta problemática, en la que vamos a profundizar más abajo, hemos de tener en cuenta que, a pesar de los cambios que introducen en sus nuevas versiones, Beller y Bender (2012) no obtienen buenos resultados en todos los casos.

En primer lugar, podemos decir que, si bien es cierto que con $\mathrm{O} 1, \mathrm{O} 2$ y $O 3$ se obtienen mejores resultados, esto es, se selecciona en mayor medida las tarjetas referentes a realizar la acción $A$ y a no cumplir la condición $P$, tal mejoría sólo lo es con respecto a los resultados obtenidos en el propio trabajo de Beller y Bender (2012) por los participantes en la versión original de Cheng y Holyoak (1985), pero no está claro que lo sea igualmente con respecto a los obtenidos en otros trabajos, como por ejemplo, sin ir más lejos, el de Beller (2010). En Beller y Bender (2012) el número de respuestas correctas en O1, O2 y $O 3$ supera el 50\%, pero no podemos olvidar que en Beller (2010) un 
58,4\% de los participantes eligió las tarjetas adecuadas en versiones deónticas abstractas similares a las de Cheng y Holyoak (1985). Evidentemente, éste es un punto que precisan aclarar Beller y Bender (2012).

$R 1, R 2$ y $R 3$ no aportan tampoco datos decisivos, pues con $R 1$ el porcentaje de selecciones válidas dista mucho de llegar al $25 \%$ y con $R 2$ y $R 3$, aunque se supera también el $50 \%$ de elecciones correctas, no se alcanzan los resultados obtenidos con $\mathrm{O} 2$ y $\mathrm{O} 3$.

$B 1$ sólo supera muy levemente el porcentaje logrado con $O 1$. No obstante, el caso de $B 2$ y $B 3$ parece ser distinto, ya que con ellas empezamos a acercarnos al 75\% de selecciones correctas. Esto, aparentemente, presta un apoyo considerable a la idea de que los seres humanos recurren a una lógica deóntica en determinadas situaciones, pero, a nuestro juicio, no es una prueba concluyente de que tal idea se sostenga, pues las explicaciones adicionales que incluyen $B 2$ y $B 3$ después de la regla propiamente dicha permiten pensar en otras posibilidades. En $B 2$ se dice con respecto a la acción $A$ que el invididuo, de otra manera, puede realizarla, mostrándose, así, con el término puede (traducción de may) la auténtica naturaleza condicional del enunciando y aclarándose que se admite que se cumpla la condición $P$ y no se realice la acción $A$, ya que, cumplida la condición $P$, la acción $A$ no tiene que realizarse forzosamente, sino que puede hacerse o no, dependiendo de, según se puede interpretar, si se desea o no. Este punto es muy importante, ya que sabemos que los condicionales pueden perfeccionarse, esto es, interpretarse como bicondicionales, lo cual lleva a considerar que sus dos miembros tienen que darse siempre unidos o no darse. Esto, para nuestro caso, significa que puede pensarse que la regla implica tanto que, si se realiza la acción $A$, hay que cumplir la condición $P$ como que, si se cumple la condición $P$, hay que realizar necesariamente la acción $A$. El fenómeno de la perfección del condicional ha sido estudiado en trabajos como, por ejemplo, Geis y Zwicky (1971), Van der Auwera (1997a, 1997b) o Moldovan (2009), pero, más allá de las explicaciones que se ofrecen en ellos sobre los posibles motivos por los que se produce, lo importante aquí es que la aclaración adicional que figura en B2 permite suponer que los participantes ejecutan adecuadamente la versión con esta regla porque tal aclaración provoca que no tiendan a perfeccionarla y que comprendan que, efectivamente, se trata de un enunciado condicional y no de otro tipo, y no porque desencadena la acción de una lógica especial de índole deóntica en la mente humana. Y algo semejante se puede decir de $B 3$, pues la utilización en ella de los verbos prohibir y permitir revela al participante, 
en virtud del contenido semántico de tales verbos, que es perfectamente admisible, según la regla, que se cumpla la condición $P$ y que, sin embargo, no se realice la acción $A$. Esto, evidentemente, también puede inhibir el fenómeno de la perfección del condicional y ayudar a entender lo que la regla verdaderamente establece. Por tanto, no puede afirmarse tampoco que los resultados de la versión con esta regla demuestren concluyentemente las tesis de los defensores de la teoría de la lógica deóntica, ya que existe igualmente una explicación alternativa para ellos.

Finalmente, hay que reconocer que los resultados con $P 1, P 2$ y $P 3$ son bastante óptimos, pero, al aparecer en $P 1$ y en $P 2$ nuevamente el término puede, cabe pensar que en ellas se hace referencia explícita otra vez a que realizar la acción $A$ es, simplemente, una posibilidad que puede concretarse o no si se ha cumplido la condición $P$, lo que significa que permiten igualmente al participante comprender el verdadero sentido del condicional y que es difícil que lo interprete en las dos direcciones, esto es, como bicondicional, y ello al margen de que $P 2$ cuenta con una explicación adicional que insiste en que, en el caso de que la condición $P$ no se cumpla, no se debe realizar la acción $A$. Por lo que se refiere a $P 3$, es obvio que los verbos permitir y prohibir pueden desempeñar la misma función, en su proceso de construcción de la representación mental correspondiente del participante, que en $B 3$, lo que se traduce en que también aclaran cuál es el verdadero significado del enunciado condicional contenido en la regla.

Evidentemente, los defensores de la lógica deóntica podrían argumentar que en los casos de $B 1, B 2, B 3, P 1, P 2$ y $P 3$ es difícil explicar los resultados apelando a capacidades como la del razonamiento lógico general de los sujetos, ya que, desde la óptica de sistemas como el de la lógica proposicional, los enunciados condicionales sólo son falsos, como se indicó más arriba, cuando el antecedente es verdadero y el consecuente es falso y en las reglas nombradas el antecedente y el consecuente están invertidos con respecto a la versión deóntica abstracta original de Cheng y Holyoak (1985). No obstante, ante esto, se puede defender, por lo que se refiere a $B 1, B 2$ y $B 3$, que, al estar ambos términos negados, la respuesta correcta sigue siendo la misma según las prescripciones lógico-formales, puesto que, siendo solamente falsa la regla cuando su antecedente es verdadero y su consecuente es falso, continúa siendo falsa solamente cuando no se cumple la condición $P$ y se realiza la acción $A$, teniendo el participante que seleccionar las mismas tarjetas que en la versión inicial de Cheng y Holyoak (1985). Por lo que respecta a P1, P2 y P3, hay 
que tener en cuenta que ya Almor y Sloman (2000) demostraron que, cuando los participantes se enfrentan a la tarea de selección, no necesariamente interpretan la regla del modo literal en el que se presenta y que, en ocasiones, invierten los términos (tomando el antecedente como consecuente y considerando al consecuente como antecedente) para que la regla sea más coherente con las instrucciones. Almor y Sloman (2000) comprobaron que esto sucede solicitando a sus participantes, después de realizar algunas versiones de la tarea, que recordaran e indicaran cuál era la regla. Está claro, desde nuestro punto de vista, que hubiera sido interesante preguntar a los participantes en las condiciones experimentales con $P 1, P 2$ y $P 3$ cuál era la regla que recordaban, pues se puede conjeturar, sin grandes esfuerzos, que, en su representación mental, el antecedente bien pudo apuntar a realizar la acción $A$ y el consecuente a cumplir la condición $P$, independientemente de lo que la regla realmente establecía.

Por tanto, creemos que, aunque la investigación de Beller y Bender (2012) constituye un esfuerzo digno de mención para probar que los seres humanos razonamos en virtud de una lógica especial en situaciones deónticas, no logra verdaderamente su propósito. Con $O 1, O 2, O 3, R 1, R 2, R 3$ y $B 1$ no se obtienen tan buenos porcentajes de selección correcta y las respuestas válidas con $B 2, B 3, P 1, P 2$ y $P 3$ son explicables en virtud de la acción de fenómenos lingüísticos o pragmáticos que pueden ser desencadenados por las características de su redacción y por los términos que aparecen en ellas.

\section{Conclusiones}

Lamentablemente, a pesar del estudio realizado por Beller y Bender (2012), continuamos sin tener certeza con respecto a lo que sucede en las versiones deónticas abstractas de la tarea de selección de las cuatro tarjetas de Peter Wason. Parece que, de alguna manera, el contenido influye en los resultados de esta tarea y el hecho de que esté planteada en términos deónticos no parece ser suficiente para aumentar los porcentajes de selección válida en ella. No podemos olvidar, en este sentido, que ni siquiera todas las versiones deónticas con contenido concreto arrojan resultados satisfactorios, como se muestra, por ejemplo, en trabajos como Cosmides et al. (2010), que, sin que ello implique que tengamos que aceptar los supuestos de los que parten, presentan importantes dificultades para la teoría de la lógica deóntica. 
Empero, no podemos descartar de manera absoluta que Beller y Bender (2012) estén en lo cierto totalmente o en algún sentido. Es perfectamente posible que las versiones deónticas de la tarea de selección que obtienen resultados discretos manifiesten problemas o dificultades en sus reglas o en sus instrucciones, pero, si ello es así, como hemos apuntado, no puede saberse a partir de sus resultados exclusivamente.

Beller y Bender (2012) tienen que explicar o explicitar, con respecto a esto, por qué los resultados de algunas de sus condiciones, como las planteadas con las reglas $\mathrm{O} 1, \mathrm{O} 2$ y $\mathrm{O3}$, no son tan óptimos como los presentan, al menos, si los comparamos con los de trabajos como el de Beller (2010), y, por supuesto, deben tratar de controlar la acción de algunas variables no consideradas, a nuestro juicio, del modo adecuado en su investigación. La presencia de determinados verbos y de aclaraciones adicionales puede permitir que el participante comprenda mejor la regla y ello, por sí mismo, ser la causa del incremento de respuestas correctas. Obviamente, tal incremento puede deberse también a los motivos señalados por Beller y Bender (2012), pero sus condiciones experimentales, tal y como están planteadas, no permiten verificarlo, pues son posibles explicaciones paralelas como las que hemos ofrecido en líneas precedentes.

Pensamos, por consiguiente, que Beller y Bender (2012) deberían, si desean demostrar sus tesis de un modo más concluyente o convincente, proponer experimentos sin explicaciones complementarias ańadidas a las reglas y sin la presencia de términos cuya carga semántica pueda transformar a las diferentes condiciones experimentales en condiciones incomparables. Mientras no procedan de esta manera, vamos, en nuestra opinión, a continuar sin contar con evidencias que nos aboquen a admitir los supuestos fundamentales de los teóricos de la lógica deóntica y, así, el problema de las versiones deónticas de la tarea de selección de las cuatro tarjetas en general y de las versiones deónticas abstractas de esa misma tarea en particular va a continuar siendo un enigma que es preciso resolver. Quizás sean necesarios nuevos procedimientos metodológicos que no se basen principalmente en el análisis cuantitativo de las respuestas de los participantes y que posibiliten adentrarse en su operar cognitivo al intentar resolver las versiones que se les presentan. Si es así, estamos ante un importante desafío para la ciencia cognitiva y para los teóricos del razonamiento que pretenden describir el funcionamiento de la actividad intelectual humana. 
En cualquier caso, no cabe duda de que esta discusión está lejos de finalizar y que los próximos estudios y las investigaciones venideras pueden abrir interesantes ámbitos de debate y proponer diferentes direcciones a las que dirigir los análisis y la experimentación. Y es que es obvio que necesitamos referencias y orientaciones diferentes a aquellas en las que nos basamos actualmente, puesto que, como se ha podido apreciar, los parámetros con los que trabajamos en el presente no nos permiten avanzar mucho más.

ASTORGA, Miguel López. Difficuties in deontic logic: the problem of abstract tasks. Trans/Form/Ação, Marília, v. 36, n. 3, p. 187-202, Set./Dez., 2013.

\begin{abstract}
The question of the existence of a deontic logic with which human beings operate in some domains must address the problem of the limited results of deontic abstract selection tasks. Recent research attempts to solve the problem by arguing that deontic abstract selection tasks have ambiguous instructions that are not easy to interpret, and by having participants work on new versions of these tasks with simple and less equivocal instructions. In this paper, I analyze the texts used in this research and the results obtained with them, and conclude that the revised approach does not really solve the problems of the initial deontic abstract tasks. I argue that the participants' answers should have been better in the new versions, and that there are pragmatic phenomena and phenomena related to textual comprehension that may have influenced the experimental results.
\end{abstract}

KEY WORDS: Conditional reasoning. Deontic logic. Pragmatics. Selection task. Textual comprehension.

\title{
REFERENCIAS
}

ALMOR, Amit; SLOMAN, Steven A. Reasoning versus text processing in the Wason selection task -a non-deontic perspective on perspective effects. Memory \& Cognition, New York, n. 28, p. 1060-1069, 2000.

AUWERA, Johan Van der. Pragmatics in the last quarter century: the case of conditional perfection. Journal of Pragmatics, Amsterdam, n. 27, p. 261-274, 1997a.

Conditional perfection. In: ATHANASIADOU, Angeliki; DIRVEN, René (Ed.). On conditionals again. Amsterdam: John Benjamins Publishing Company, 1997b. p. 169-190.

BELLER, Sieghard. Deontic reasoning reviewed: psychological questions, empirical findings, and current theories. Cognitive Processing, New York, n. 11, p. 123-132, 2010. 
; BENDER, Andrea. Competent deontic reasoning: the abstract deontic selection task revisited. In: MIYAKE, Naomi; PEEBLES, David; COOPER, Richard P. (Ed.). ANNUAL CONFERENCE OF THE COGNITIVE SCIENCE SOCIETY, $34^{\text {th }}$. Proceedings... Austin, Texas: Cognitive Science Society, 2012, p. 114-119.

CHENG, Patricia W.; HOLYOAK, Keith J. Pragmatic reasoning schemas. Cognitive Psychology, Amsterdam, n. 17, p. 391-416, 1985.

; HOLYOAK, Keith J. On the natural selection of reasoning theories. Cognition, Amsterdam, n. 33, p. 285-313, 1989.

COSMIDES, Leda. The logic of social exchange: has natural selection shaped how humans reason? Studies with the Wason selection task. Cognition, Amsterdam, n. 31, p. 187-276, 1989.

; BARRETT, H. Clark; TOOBY, John. Adaptive specializations, social exchange, and the evolution of human intelligence. Proceedings of the National Academy of Sciences, Washington, n. 107, p. 9007-9014, 2010.

; TOOBY, John. Cognitive adaptations for social exchange. In: BARKOW, Jerome; COSMIDES, Leda; J. TOOBY, John (Ed.). The adapted mind: evolutionary psychology and the generation of culture. New York: Oxford University Press, 1992, p. 163-228.

COX, James R.; GRIGGS, Richard A. The effect of experience on performance in Wason's selection task. Memory \& Cognition, New York, n. 10, p. 496-502, 1982.

FIDDICK, Laurence; COSMIDES, Leda; TOOBY, John. No interpretation without representation: the role of domain-specific representations and inferences in the Wason selection task. Cognition, Amsterdam, n. 77, p. 1-79, 2000.

; ERLICH, Nicole. Giving it all away: altruism and answers to the Wason selection task. Evolution and Human Behavior, Amsterdam, n. 31, p. 131-140, 2010.

GEIS, Michael L.; ZWICKY, Arnold M. On invited inferences. Linguistic Inquiry. Cambridge, Massachusetts, n. 2, p. 561-566, 1971.

MOLDOVAN, Andrei. Pragmatic considerations in the interpretation of denying the antecedent. Informal Logic, Windsor, v. 29, n. 3, p. 309-326, 2009.

WASON, Peter C. Reasoning. In: FOSS, Brian M. (Comp.). New horizons in psychology. Harmondsworth, Middlesex: Penguin, 1966, p. 135-151.

. Reasoning about a rule. Quarterly Journal of Experimental Psychology, London, $\mathrm{n}$. 20, p. 273-281, 1968.

Recebido em: 27.10.2012

Aceito em: 22.01.2013 